\title{
ASYMPTOTIC ANALYSIS OF A DYNAMICAL SYSTEM ARISING IN THERMOELASTIC CONTACT*
}

\author{
P. D. HOWELL ${ }^{\dagger}$
}

\begin{abstract}
We consider a simple dynamical model for a discrete system of $N$ metal pins in frictional thermoelastic contact with a moving surface. In general, the model consists of $N$ heat equations for the temperature in each pin, subject to nonsmooth and nonlocal coupling through the conditions for contact between each pin and the moving surface. When the normalized coefficient of thermal expansion is sufficiently large, the model predicts large-amplitude oscillatory behavior in qualitative agreement with experimental observations of thermoelastic instability. We use perturbation methods to analyze the model and thus describe in detail the bifurcation behavior of the system as the relevant physical parameters are varied.
\end{abstract}

Key words. thermoelastic instability, dynamical systems, asymptotic analysis

AMS subject classifications. 37N15, 41A60, 74F05, 74M10, 74M15

DOI. $10.1137 / 18 \mathrm{M} 1191191$

1. Introduction. Mechanical systems where one elastic solid slides over another often suffer from the so-called thermoelastic instability (TEI), resulting in harmful localized heating and oscillations, referred to as "hot-spotting" and "hot judder" in applications such as braking systems [3, 19]. TEI was first identified and analyzed by Barber [6], who also studied a simplified physical model that illustrates the main underlying physical mechanisms of the instability. Barber's [6] model system consists of $N$ metal pins (with $N=3$ in the original experiments), pressed against a surface that moves tangentially at constant speed. The sliding motion causes frictional heating and thus thermal expansion, which acts to increase the pin length, but also frictional wear, which acts to decrease the pin length. In regimes such that thermal expansion dominates, Barber [6] observed oscillations characterized by long periods where just one pin is in contact with the moving surface, punctuated by very rapid transitions from one pin to the next.

A simple mathematical model for the case of $N=2$ pins was derived by Ockendon and Barber [13], and preliminary numerical simulations in [2] demonstrated that the model can indeed produce oscillations in qualitative agreement with those observed in Barber's [6] experiments. In this paper we perform a detailed analysis of the twopin model from [13] which both allows the observed nonlinear oscillatory behavior to be explained and quantified, and uncovers hitherto unsuspected bifurcations and hysteresis. The generalization to $N=3$ or more pins opens up the possibility of even more exotic behavior, as shown in [11].

The contact problem between each pin and the tangentially sliding surface gives rise to a nonsmooth and nonlocal relation between the pin lengths and the compressive forces in the pins. Similar nonsmooth behavior can arise for example in impact problems [9] and in stick-slip models [15, 20], and is often described using so-called Filippov systems and differential inclusions $[8,10,16]$. We show that the apparently discontinuous behavior can be unfolded by a judicious choice of dependent variables,

* Received by the editors May 30, 2018; accepted for publication (in revised form) October 8 , 2018; published electronically November 27, 2018.

http://www.siam.org/journals/siap/78-6/M119119.html

${ }^{\dagger}$ Mathematical Institute, Andrew Wiles Building, Oxford OX2 6GG, UK (howell@maths.ox. ac.uk). 
resulting in a nonsmooth continuous system rather than a Filippov system, in the language of [12].

This paper is organized as follows. In section 2 we present the general mathematical model for the $N$-pin problem before restricting ourselves to the case of $N=2$ pins; further details of the derivation of the model are given in Appendix A. In section 3 we consider solutions that are periodic in time and show that the problem can then be recast as an infinite system of algebraic equations. In section 4 we perform a formal asymptotic analysis of the system in the regime where thermal expansion dominates. We are thus able to quantify how the period and amplitude of the oscillations, and the rate of load transfer between the pins, all depend on the physical parameters in the model. Finally, in section 5 we discuss our results and draw our conclusions.

\section{Mathematical model.}

2.1. General formulation. The system under consideration involves $N$ metal pins, each with its base clamped to a metal arm, while the other end is pressed with force $F_{j}(j=1, \cdots, N)$ against a surface moving tangentially at constant speed. The resulting frictional contact causes heating of the pin and also wear, both at a rate proportional to the contact force $F_{j}$. A derivation of the following dimensionless model is given in [11] and briefly summarised in Appendix A.

The temperature $T_{j}(X, t)$ in the $j$ th pin satisfies the boundary-value problem

$$
\begin{aligned}
\frac{\partial^{2} T_{j}}{\partial X^{2}} & =\frac{\partial T_{j}}{\partial t}, & & 0<<1, \\
\frac{\partial T_{j}}{\partial X} & =h T_{j}, & X & =0, \\
\frac{\partial T_{j}}{\partial X} & =F_{j}, & X & =1,
\end{aligned}
$$

where $X=1$ refers to the end of the pin in frictional contact with the moving surface, and $X=0$ refers to the base of the pin, where there is an effective heat transfer coefficient $h$. The net strain $d_{j}$ of the $j$ th pin satisfies the differential equation

$$
\frac{\mathrm{d}}{\mathrm{d} t}\left(F_{j}+d_{j}\right)=(\alpha-\eta) F_{j}-\left.\alpha \frac{\partial T_{j}}{\partial X}\right|_{X=0},
$$

where $\alpha$ and $\eta$ are two dimensionless parameters measuring the effects of thermal expansion and frictional wear, respectively.

The problem is closed by considering the state of contact between each pin and the moving surface. The normal force $F_{j}$ cannot be negative, and is only nonzero when pin $j$ is in contact; on the other hand, all pins that are in contact must have equal lengths, and must be longer than any pins that are out of contact. These constraints may be combined in a set of complementarity conditions of the form

$$
\begin{aligned}
\sum_{i} F_{i} & =1, \\
F_{j} \geq 0, \quad\left(\max _{i}\left(d_{i}\right)-d_{j}\right) F_{j} & =0 \quad \text { for } j=1, \cdots, N .
\end{aligned}
$$

Given suitable initial conditions for $T_{j}(X, 0), F_{j}(0)$, and $d_{j}(0),(2.1)-(2.3)$ in principle determine $T_{j}(X, t), F_{j}(t)$, and $d_{j}(t)$ for $t>0$. 
The solution of the boundary-value problem (2.1) in principle allows the flux term in (2.2) to be expressed as a linear functional of the force $F_{j}$, i.e.,

$$
\left.\frac{\partial T_{j}}{\partial X}\right|_{X=0}=\int_{0}^{t} F_{j}(\tau) G(t-\tau) \mathrm{d} \tau=Q\left[F_{j}\right](t) .
$$

By taking a Laplace transform, we can explicitly solve for the relevant Green's function (which depends parametrically on the coefficient $h$ ) in the form

$$
G(h ; t)=\frac{1}{2 \pi \mathrm{i}} \int_{\epsilon-\mathrm{i} \infty}^{\epsilon+\mathrm{i} \infty} K(h, \sqrt{p}) \mathrm{e}^{p t} \mathrm{~d} p,
$$

where $\epsilon>0$ and, for future reference, we have introduced the function

$$
K(h, s):=\frac{1}{\cosh s+(s / h) \sinh s} .
$$

By summing over the poles in $K$, we can alternatively express $G$ as the infinite series

$$
G(h ; t)=\sum_{n=0}^{\infty} \frac{2 h^{2} \cos \left(k_{n}(h)\right)}{h+\sin ^{2}\left(k_{n}(h)\right)} \mathrm{e}^{-k_{n}(h)^{2} t},
$$

where $k_{n}(h)$ is the root in the range $n \pi<k<(n+1 / 2) \pi$ of the transcendental equation $k \tan k=h$. The formulation (2.4) allows the temperature $T_{j}$ to be eliminated from the problem completely, turning the remaining equations (2.2) and (2.3) into a system of algebraic and integro-differential equations for $F_{j}$ and $d_{j}$.

2.2. The two-pin problem. In this paper we will focus on the case $N=2$, in which case the problem can be expressed purely in terms of the differences between the temperatures, forces, and displacements in each of the two pins. To this end, we define

$$
T_{1}(X, t)-T_{2}(X, t)=\theta(X, t), \quad F_{1}(t)-F_{2}(t)=\Lambda(t), \quad d_{1}(t)-d_{2}(t)=U(t) .
$$

In terms of $\Lambda$ and $U$, the complementarity condition (2.3) becomes

$$
\left(1-\Lambda^{2}\right) U=0, \quad(1+\Lambda) U \geq 0, \quad(1-\Lambda) U \leq 0, \quad\left(1-\Lambda^{2}\right) \geq 0 .
$$

The inequalities in (2.9) define the set in the $(U, \Lambda)$-plane illustrated in Figure 1(a). It is more convenient instead to pose the problem in terms of the quantity

$$
H=\Lambda+U,
$$

so that (2.9) defines $\Lambda$ as a continuous function of $H$, namely,

$$
\Lambda(H)=\frac{1}{2}(|H+1|-|H-1|),
$$

as plotted in Figure 1(b).

From $(2.1)$, we find that $\theta(X, t)$ satisfies the boundary-value problem

$$
\begin{aligned}
\frac{\partial^{2} \theta}{\partial X^{2}}=\frac{\partial \theta}{\partial t}, & & <X<1, \\
\frac{\partial \theta}{\partial X}=h \theta, & X & =0, \\
\frac{\partial \theta}{\partial X}=\Lambda(H), & X & =1,
\end{aligned}
$$

Copyright $@$ by SIAM. Unauthorized reproduction of this article is prohibited. 


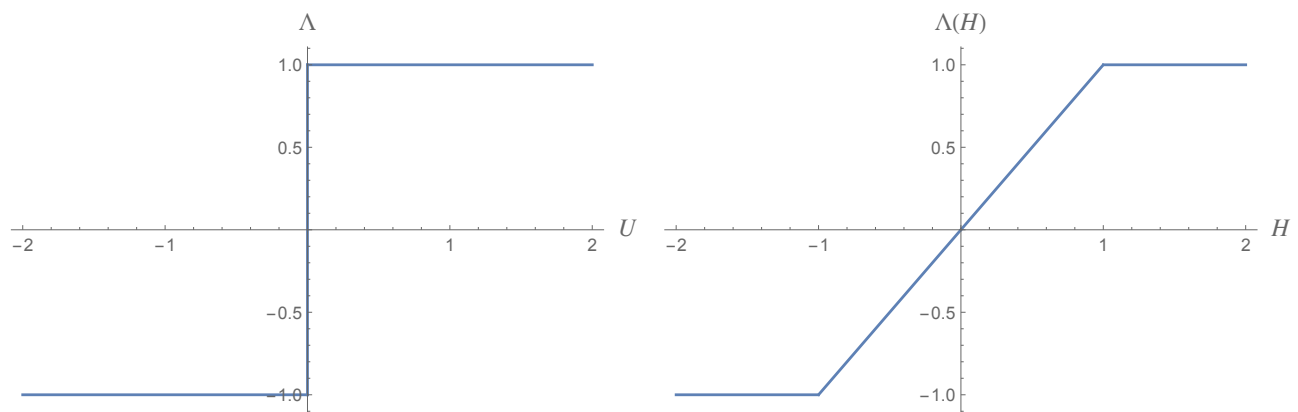

(a)

(b)

FIG. 1. (a) The relation between $\Lambda$ and $U$ implied by the inequalities (2.9). (b) The function $\Lambda(H)$ defined by (2.11).

and the evolution equation (2.2) leads to

$$
\frac{\mathrm{d} H}{\mathrm{~d} t}-(\alpha-\eta) \Lambda(H)+\left.\alpha \frac{\partial \Theta}{\partial X}\right|_{X=0}=0 .
$$

In terms of the functional $Q$ introduced in (2.4), we can reduce (2.12) to a single equation for the function $H(t)$, namely,

$$
\frac{\mathrm{d} H}{\mathrm{~d} t}-(\alpha-\eta) \Lambda(H)+\alpha Q[\Lambda(H)]=0 .
$$

Given a suitable initial condition for $H(0),(2.13)$ provides a closed problem for $H(t)$, with the function $\Lambda(H)$ defined by (2.11). The reduction of the infinite-dimensional problem (2.12) to the scalar dynamical system (2.13), driven by the nonsmooth forcing function $\Lambda(H)$ and a history integral of the same forcing function, echoes a similar dimensional reduction employed in $[17,18]$ to describe nonsmooth forcing due to friction and impact in point contact between elastic bodies.

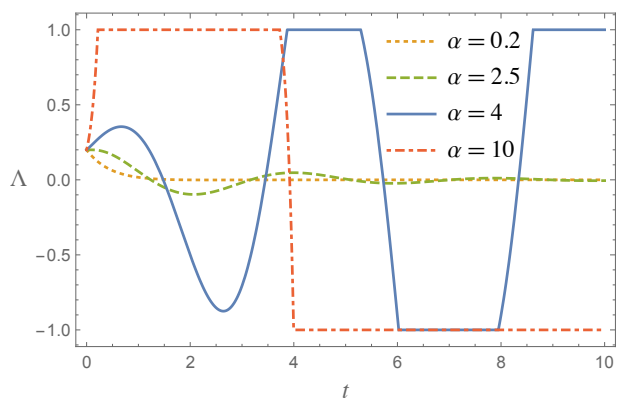

(a)

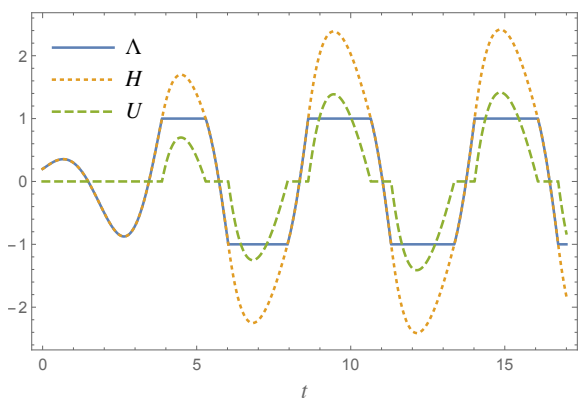

(b)

FIG. 2. (a) Numerical solutions of the 2-pin problem (2.13) computed using the initial condition $H(0)=0.2$, with $h=2, \eta=2.5$, and various values of $\alpha$. (b) Results of numerical solution of (2.13) with $h=2, \eta=2.5, \alpha=4$, and $H(0)=0.2$; the three curves show the solution $H(t)$, the force difference $\Lambda(H(t))$, and the strain difference $U(t)=H(t)-\Lambda(H(t))$, respectively.

Copyright (c) by SIAM. Unauthorized reproduction of this article is prohibited. 
2.3. Numerical results. In Figure 2 we show some typical numerical solutions of (2.13), subject to the initial condition $H(0)=0.2$. In Figure $2(a)$ we fix the values of $h=2$ and $\eta=2.5$, while varying the value of $\alpha$, and plot the solutions for $\Lambda(H)$ versus $t$. In Figure 2(b), we show the behaviors of $H(t), \Lambda(H(t))$, and $U(t)=H(t)-\Lambda(H(t))$ with parameter values $h=2, \eta=2.5, \alpha=4$, and initial condition $H(0)=0.2$; the blue solid graphs for $\Lambda$ shown in Figures 2(a) and 2(b) are identical. We recall that $\Lambda$ represents the difference between the forces while $U$ represents the difference between the strains in the two pins, and the plots may therefore be interpreted as follows. Whenever $\Lambda$ is between -1 and +1 , both of the pins are in contact and sharing the load, and $U$ is therefore zero. When $\Lambda$ reaches its maximum value of +1 , only pin 1 is in contact, which implies that pin 1 is longer than pin 2 and hence $U>0$. Conversely, when $\Lambda=-1$, only pin 2 is in contact and therefore $U<0$.

For the smaller values of $\alpha$ shown in Figure 2(a), the solution for $\Lambda$ decays exponentially to zero, but at larger values of $\alpha$, the system instead undergoes nonlinear oscillations, with the two pins taking turns to support the applied load, and the same qualitative behavior is observed for other values of $h$ and $\eta$. One can understand the transition between these two types of behavior by analysing the linear stability of the trivial solution $H \equiv 0$, corresponding to a uniform equilibrium state, having both pins in contact with equal lengths and temperatures. Nontrivial small perturbations of the form $H(t) \propto \mathrm{e}^{\sigma t}$ are consistent with (2.13) provided the linear growth rate $\sigma$ satisfies the dispersion relation

$$
\sigma+\eta-\alpha+\alpha K(h, \sqrt{\sigma})=0,
$$

where $K$ is again the function defined in (2.6). If the values of $h$ and $\eta$ are fixed then, as $\alpha$ increases through some critical value, the dominant root $\sigma$ of (2.14) with the largest real part crosses the imaginary axis, and the uniform state $H=0$ loses stability. The details may be found in [11], and the stability boundary in the $(\alpha, \eta)$ plane when $h=2$ is shown in Figure 3. For our purposes, it is germane to note that the boundary approaches the linear behavior $\eta / \alpha \rightarrow \zeta_{*}(h)$ as $\alpha \rightarrow \infty$, where the limiting gradient $\zeta_{*}$ is a function of $h$ that lies in the range $1<\zeta_{*}(h)<1+\operatorname{sech} \pi \approx 1.086$.

Since $\Lambda(H) \equiv H$ when $|H|<1$, the problem (2.13) is linear when $|H|<1$. Whenever the base state is unstable, any perturbations continue to grow exponentially until $H$ reaches \pm 1 , and the system then undergoes oscillations in which the contact repeatedly switches between one pin and the other. The nonsmoothness of the function $\Lambda$ thus results in a nongeneric Hopf bifurcation, with a finite-amplitude limit cycle appearing immediately as the stability boundary depicted in Figure 3 is crossed.

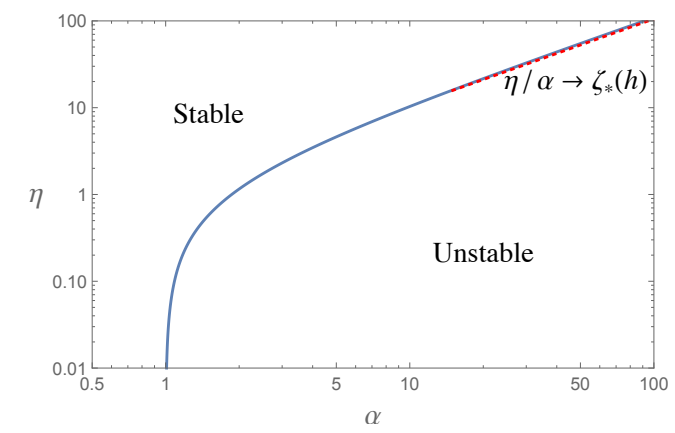

FIG. 3. The stability boundary in the $(\alpha, \eta)$-plane with $h=2$. The red dotted curve indicates the limiting behavior $\eta / \alpha \rightarrow \zeta_{*}(2) \approx 1.054$ as $\alpha \rightarrow \infty$. 
The graphs of $\Lambda$ and $U$ versus $t$ in Figure 2(b) both exhibit slope discontinuities at values of $t$ where load is transferred, corresponding to a switch in the active inequalities in the complementarity condition (2.9). In contrast, the auxiliary function $H(t)$ is everywhere differentiable. This fact partly explains why it is sensible to use $H$ as the main dependent variable rather than $\Lambda$ or $U$, despite it being a mathematical construct with no obvious physical interpretation. As the value of $\alpha$ increases, the amplitude of $H(t)$ increases also, while $\Lambda$ is constrained between the values of \pm 1 . The upshot is that the oscillations become increasingly violent, and the system spends the majority of the time with just one pin in contact, with the switches in contact between the two pins (i.e., between $\Lambda=-1$ and $\Lambda=+1$ ) occurring extremely rapidly. In what follows, we analyze this behavior by seeking periodic solutions of (2.13) and then taking the formal asymptotic limit $\alpha \rightarrow \infty$.

\section{Periodic solutions.}

3.1. The full problem. Suppose that, after some transient, the system has settled down to a periodic solution of period $2 \tau$, with typical behaviors of $H(t)$ and $\Lambda(H(t))$ as shown schematically in Figure 4 . Without loss of generality we choose the origin for $t$ such that $H(0)=0$. Since (2.13) is invariant under the transformations $t \mapsto t+$ const and $H \mapsto-H$, we infer that periodic solutions satisfy $H(t+\tau) \equiv-H(t)$. Therefore the dependent variables may be expressed as Fourier series of the forms

$$
H(t)=\sum_{\substack{n=-\infty \\ n \text { odd }}}^{\infty} b_{n} \mathrm{e}^{\mathrm{i} n \pi t / \tau}, \quad \Lambda(H(t))=\sum_{\substack{n=-\infty \\ n \text { odd }}}^{\infty} c_{n} \mathrm{e}^{\mathrm{i} n \pi t / \tau},
$$

where $b_{-n} \equiv \overline{b_{n}}$ and $c_{-n} \equiv \overline{c_{n}}$. Henceforth, we assume implicitly that $b_{n}$ and $c_{n}$ are identically zero when $n$ is even and so we may drop the restriction " $n$ odd" from the summation signs.

After substituting (3.1) into the system (2.12), we can solve for $\theta(X, t)$ and thus calculate

$$
Q[\Lambda]=\left.\frac{\partial \theta}{\partial X}\right|_{X=0}=\sum_{n=-\infty}^{\infty} K_{n}(h, \tau) c_{n} \mathrm{e}^{\mathrm{i} n \pi t / \tau}
$$

where we have introduced the shorthand

$$
K_{n}=K_{n}(h, \tau):=K\left(h, \sqrt{\frac{\mathrm{i} n \pi}{\tau}}\right),
$$

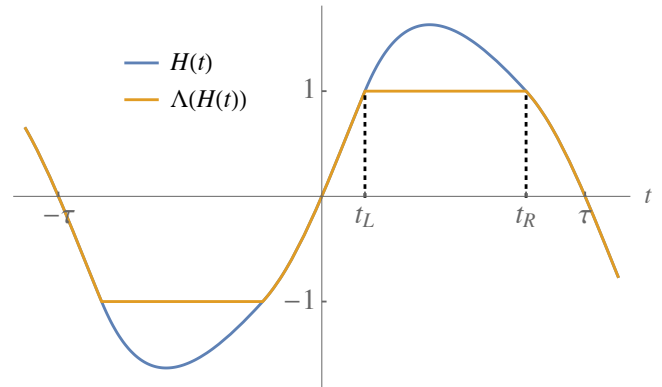

FIG. 4. Schematic of the behaviors of $H(t)$ and $\Lambda(H(t))$ in a periodic solution with period $2 \tau$. 
and $K$ is again the function defined by (2.6). The evolution equation (2.13) therefore implies the following relation between the coefficients $b_{n}$ and $c_{n}$ :

$$
\frac{\mathrm{i} n \pi}{\tau} b_{n}=\left(\alpha-\eta-\alpha K_{n}\right) c_{n}
$$

A second relation between the coefficients may be found by noting that $\Lambda(H)=H$, when $0 \leq H \leq 1$ and $\Lambda(H)=1$ when $H>1$. We can therefore write

$$
c_{n}=\frac{1}{\tau}\left(\int_{0}^{t_{L}}+\int_{t_{R}}^{\tau}\right) H(t) \mathrm{e}^{-\mathrm{i} n \pi t / \tau} \mathrm{d} t+\frac{1}{\tau} \int_{t_{L}}^{t_{R}} \mathrm{e}^{-\mathrm{i} n \pi t / \tau} \mathrm{d} t,
$$

where $t_{L}$ and $t_{R}$ are the two values of $t$ in the range $0<t_{L}<t_{R}<\tau$ at which $H(t)$ passes through the value 1, as illustrated in Figure 4. By evaluating the right-hand side of (3.5), we obtain a system of linear equations for the coefficients $b_{n}$, namely,

$$
\begin{aligned}
c_{n} & =\frac{\mathrm{i} n \pi}{\tau\left(\alpha-\eta-\alpha K_{n}\right)} b_{n}=\frac{\mathrm{i}}{n \pi}\left(\mathrm{e}^{-\mathrm{i} n \pi t_{R} / \tau}-\mathrm{e}^{-\mathrm{i} n \pi t_{L} / \tau}\right) \\
& +\left(\frac{t_{L}+\tau-t_{R}}{\tau}\right) b_{n}+\frac{\mathrm{i}}{\pi} \sum_{n \neq m=-\infty}^{\infty} \frac{b_{m}}{m-n}\left[\mathrm{e}^{\mathrm{i}(m-n) \pi t_{R} / \tau}-\mathrm{e}^{\mathrm{i}(m-n) \pi t_{L} / \tau}\right] .
\end{aligned}
$$

To close the problem, we impose the conditions $H(0)=0$ and $H\left(t_{L}\right)=H\left(t_{R}\right)=1$ to obtain

$$
\sum_{n=-\infty}^{\infty} b_{n}=\sum_{n=-\infty}^{\infty} b_{n} \mathrm{e}^{\mathrm{i} n \pi t_{L} / \tau}-1=\sum_{n=-\infty}^{\infty} b_{n} \mathrm{e}^{\mathrm{i} n \pi t_{R} / \tau}-1=0
$$

In principle, the linear system (3.6) can be solved for the coefficients $b_{n}$, and then the remaining unknowns $t_{L}, t_{R}$, and $\tau$ can be determined from the three supplementary equations (3.7).

3.2. Behavior as $n \rightarrow \infty$. Before proceeding with our asymptotic analysis of the now discrete problem (3.6) and (3.7), we first check the convergence of the series by examining the behaviors of $b_{n}$ and $c_{n}$ as $n \rightarrow \infty$. No generality is lost by taking $n>0$ since the corresponding behavior of $b_{-n}$ and $c_{-n}$ may be found by conjugating.

By using (3.7), we can simplify (3.6) to

$$
\begin{aligned}
& {\left[\frac{\mathrm{i} n \pi}{\tau\left(\alpha-\eta-\alpha K_{n}\right)}-\frac{t_{L}+\tau-t_{R}}{\tau}\right] b_{n}} \\
& \quad=\frac{\mathrm{i}}{n \pi} \sum_{n \neq m=-\infty}^{\infty} \frac{m b_{m}}{m-n}\left[\mathrm{e}^{\mathrm{i}(m-n) \pi t_{R} / \tau}-\mathrm{e}^{\mathrm{i}(m-n) \pi t_{L} / \tau}\right] .
\end{aligned}
$$

When $n$ is large, $K_{n}$ is exponentially small, and we may also expand the right-hand side of (3.8) to obtain

$$
\begin{aligned}
{\left[\frac{\mathrm{i} n \pi}{\tau(\alpha-\eta)}-\frac{t_{L}+\tau-t_{R}}{\tau}\right] b_{n} \sim } & -\frac{\mathrm{i}}{n^{2} \pi} \sum_{n \neq m=-\infty}^{n-2 M} m b_{m}\left[\mathrm{e}^{\mathrm{i}(m-n) \pi t_{R} / \tau}-\mathrm{e}^{\mathrm{i}(m-n) \pi t_{L} / \tau}\right] \\
& +\frac{\mathrm{i}}{2 n \pi} \sum_{0 \neq j=1-M}^{\infty} \frac{(n+2 j) b_{n+2 j}}{j}\left[\mathrm{e}^{2 \mathrm{i} j \pi t_{R} / \tau}-\mathrm{e}^{2 \mathrm{ij} \pi t_{L} / \tau}\right],
\end{aligned}
$$

where $1 \ll M \ll n$. 
As illustrated in Figure 4, we hypothesize that $\Lambda(H(t))$ is continuous and $H(t)$ is differentiable, so that $c_{n}=O\left(n^{-2}\right)$ and $b_{n}=O\left(n^{-3}\right)$ as $n \rightarrow \infty$. It follows that the second sum in (3.9) is $O\left(n^{-3}\right)$, and thus to lowest order we obtain

$$
b_{n} \sim \frac{\mathrm{i}(\alpha-\eta) \tau}{n^{3} \pi^{2}}\left[\mathrm{e}^{-\mathrm{i} n \pi t_{R} / \tau} \dot{H}\left(t_{R}\right)-\mathrm{e}^{-\mathrm{i} n \pi t_{L} / \tau} \dot{H}\left(t_{L}\right)\right] \quad \text { as } n \rightarrow \infty,
$$

where

$$
\dot{H}(t)=\sum_{m=-\infty}^{\infty} \mathrm{i} m b_{m} \mathrm{e}^{\mathrm{i} m \pi t / \tau}
$$

Thus we indeed find that $b_{n}=O\left(n^{-3}\right)$ as $n \rightarrow \infty$, in agreement with our hypothesis.

\section{Large- $\alpha$ limit.}

4.1. Leading-order problem. We are interested in violent oscillations, such as those shown by the solid blue curves in Figure 2, where the graph of $\Lambda(H(t))$ resembles a square wave, corresponding to long periods in which just one pin is in contact and very rapid transitions between the two pins. We anticipate that such behavior occurs when $\alpha$ is large, and we will therefore consider the asymptotic limit $\alpha \rightarrow \infty$ while treating the parameter $\zeta=\eta / \alpha$ as $O(1)$. We expect the amplitude of $H(t)$ to be large in this limit, while $\Lambda$ remains $O(1)$, and the values of $t_{L}$ and $t_{R}$ consequently become close to 0 and $\tau$, respectively. We therefore rescale as follows:

$$
b_{n}=\alpha B_{n}, \quad t_{L}=\frac{\tau}{\alpha \pi} \xi_{L}, \quad t_{R}=\tau-\frac{\tau}{\alpha \pi} \xi_{R},
$$

where $B_{n}$ and $\xi_{L, R}$ are $O(1)$. The analysis of subsection 3.2 implies that $b_{n}=$ $O\left(\alpha^{2} / n^{3}\right)$ as $n \rightarrow \infty$. In particular, let us suppose that $b_{n}$ takes the form

$$
b_{n} \sim \frac{\alpha^{2}}{n^{3}} g\left(\frac{n}{\alpha}\right) \quad \text { when } n=O(\alpha) \gg 1,
$$

where the function $g$ is $O(1)$.

In terms of the rescaled variables, (3.6) takes the form

$$
\begin{aligned}
& \frac{\mathrm{i} n \pi}{\left(1-\zeta-K_{n}\right) \tau} B_{n}=-\frac{\mathrm{i}}{n \pi}\left(\mathrm{e}^{\mathrm{i} n \xi_{R} / \alpha}+\mathrm{e}^{-\mathrm{i} n \xi_{L} / \alpha}\right) \\
& \quad+\left(\frac{\xi_{L}+\xi_{R}}{\pi}\right) B_{n}+\frac{\mathrm{i} \alpha}{\pi} \sum_{n \neq m=-\infty}^{\infty} \frac{B_{m}}{m-n}\left[\mathrm{e}^{-\mathrm{i}(m-n) \xi_{R} / \alpha}-\mathrm{e}^{\mathrm{i}(m-n) \xi_{L} / \alpha}\right] .
\end{aligned}
$$

We first expand the right-hand side in the limit $\alpha \rightarrow \infty$ with $n=O(1)$ by splitting up the sum as follows:

$$
\begin{aligned}
& \sum_{n \neq m=-\infty}^{\infty} \frac{B_{m}}{m-n}\left[\mathrm{e}^{-\mathrm{i}(m-n) \xi_{R} / \alpha}-\mathrm{e}^{\mathrm{i}(m-n) \xi_{L} / \alpha}\right] \\
& =\sum_{n \neq m=-M}^{M} \frac{B_{m}}{m-n}\left[\mathrm{e}^{-\mathrm{i}(m-n) \xi_{R} / \alpha}-\mathrm{e}^{\mathrm{i}(m-n) \xi_{L} / \alpha}\right] \\
& \quad+\frac{1}{\alpha^{3}}\left[\sum_{m=-\infty}^{-M-2}+\sum_{m=M+2}^{\infty}\right] \frac{g\left(k_{m}\right)}{k_{m}^{3}\left(k_{m}-n / \alpha\right)}\left[\mathrm{e}^{-\mathrm{i}\left(k_{m}-n / \alpha\right) \xi_{R}}-\mathrm{e}^{\mathrm{i}\left(k_{m}-n / \alpha\right) \xi_{L}}\right]
\end{aligned}
$$

Copyright $@$ by SIAM. Unauthorized reproduction of this article is prohibited. 
with $k_{m}:=m / \alpha$ and $1 \ll M \ll \alpha$. In the first series on the right-hand side of (4.4), the exponentials may be expanded using the largeness of $\alpha$, while the final two series may be approximated using Euler-Maclaurin summation [14]. We thus find that (4.3) takes the form

$$
\begin{aligned}
& \frac{\mathrm{i} n \pi}{\left(1-\zeta-K_{n}\right) \tau} B_{n}+\frac{2 \mathrm{i}}{n \pi} \\
& \quad \sim \frac{\left(\xi_{L}+\xi_{R}\right)}{\pi} \sum_{m=-M}^{M} B_{m}+\frac{\mathrm{i}\left(\xi_{L}^{2}-\xi_{R}^{2}\right) g(0)}{2 \pi M}+\frac{\left(\xi_{L}+\xi_{R}\right) g^{\prime}(0)}{\pi M}+O\left(\alpha^{-1}\right)
\end{aligned}
$$

as $\alpha \rightarrow \infty$. Now letting $M \rightarrow \infty$, we see that the right-hand side of (4.5) tends to zero by virtue of (3.7).

Thus we obtain the leading-order approximation

$$
B_{n}=-\frac{2\left(1-\zeta-K_{n}\right) \tau}{\pi^{2} n^{2}}
$$

valid in the limit as $\alpha \rightarrow \infty$ with $n=O(1)$. Since $K_{n}$ is exponentially small for large $n$, we have $B_{n} \sim-2(1-\zeta) \tau /\left(\pi^{2} n^{2}\right)$ as $n \rightarrow \infty$, and by asymptotic matching with the large- $n$ ansatz (4.2) we deduce that the function $g$ must satisfy

$$
g(k) \sim-\frac{2(1-\zeta) \tau}{\pi^{2}} k \quad \text { as } k \rightarrow 0 .
$$

In particular, we will exploit the fact that $g(0)=0$ to simplify the analysis henceforth.

When considering the behavior as $\alpha \rightarrow \infty$ with $k=n / \alpha=O(1)$, it is more convenient to start from the equivalent system (3.8) rather than (3.6). The key again is to split up the series on the right-hand of (3.8) in the same manner as above, and the result is an integral equation for the function $g$, namely,

$$
\frac{\pi^{2}}{(1-\zeta) \tau} g(k)=\mathrm{i}\left(\mathrm{e}^{\mathrm{i} k \xi_{R}}-\mathrm{e}^{-\mathrm{i} k \xi_{L}}\right) S_{1}+\frac{1}{2} f_{-\infty}^{\infty} \frac{k g(\ell)}{(\ell-k) \ell^{2}}\left(\mathrm{e}^{\mathrm{i}(k-\ell) \xi_{R}}-\mathrm{e}^{\mathrm{i}(\ell-k) \xi_{L}}\right) \mathrm{d} \ell .
$$

The principal value integral on the right-hand side of (4.8) exists by virtue of (4.7). The notation $S_{1}$ is shorthand for the leading-order approximation to $\dot{H}(0)$, namely,

$$
S_{1}:=\mathrm{i} \sum_{n=-\infty}^{\infty} n B_{n}
$$

where the dashed sum notation denotes the principal value

$$
\sum_{n=-\infty}^{\infty} c_{n}:=\lim _{M \rightarrow \infty} \sum_{n=-M}^{M} c_{n} .
$$

Finally, we use the same approach to expand the three constraints (3.7) in the limit $\alpha \rightarrow \infty$, resulting in

$$
\sum_{n=-\infty}^{\infty} B_{n}=0
$$

Copyright $@$ by SIAM. Unauthorized reproduction of this article is prohibited. 


$$
2 \xi_{L} S_{1}=2+f_{-\infty}^{\infty} \frac{g(k)\left(1-\mathrm{e}^{\mathrm{i} \xi_{L} k}\right)}{k^{3}} \mathrm{~d} k, \quad 2 \xi_{R} S_{1}=2+f_{-\infty}^{\infty} \frac{g(k)\left(\mathrm{e}^{-\mathrm{i} \xi_{R} k}-1\right)}{k^{3}} \mathrm{~d} k,
$$

where $S_{1}$ again refers to the series (4.9). In passing, we note that (4.12) may be used to show that the right-hand side of (4.8) looks like $-2 k$ as $k \rightarrow 0$, in agreement with (4.7).

By substituting the leading-order expression (4.6) for $B_{n}$ into (4.9) and (4.11), we obtain

$$
\begin{aligned}
\zeta & =1-\frac{4}{\pi^{2}} \sum_{n=-\infty}^{\infty} \frac{1}{n^{2}} K\left(h, \sqrt{\frac{\mathrm{i} n \pi}{\tau}}\right), \\
S_{1} & =\frac{2 \mathrm{i} \tau}{\pi^{2}} \sum_{n=-\infty}^{\infty} \frac{1}{n} K\left(h, \sqrt{\frac{\mathrm{i} n \pi}{\tau}}\right) .
\end{aligned}
$$

Thus the period $\tau$, and then the series $S_{1}$, are in principle determined in terms of the material parameters $\zeta$ and $h$. It remains to determine $\xi_{L}$ and $\xi_{R}$ from (4.12), to which end we next solve the integral equation (4.8) for the function $g(k)$.

4.2. Solution of integral equation. Let us define

$$
\hat{h}(k)=\frac{g(k)}{k^{2}}-\frac{g^{\prime}(0)}{k},
$$

so that $\hat{h}(k)$ is regular as $k \rightarrow 0$. Here and henceforth we use the hat notation to denote the Fourier transform, defined such that

$$
\hat{h}(k)=\int_{-\infty}^{\infty} h(t) \mathrm{e}^{-\mathrm{i} k t} \mathrm{~d} t, \quad h(t)=\frac{1}{2 \pi} \int_{-\infty}^{\infty} \hat{h}(k) \mathrm{e}^{\mathrm{i} k t} \mathrm{~d} k .
$$

Then (4.12) may be written in the form

$$
\begin{aligned}
& \left(2 S_{1}-\pi g^{\prime}(0)\right) \xi_{L}=2-\mathrm{i} \int_{-\infty}^{\infty} \hat{h}(k) \hat{\chi}_{\left[0, \xi_{L}\right]}(-k) \mathrm{d} k \\
& \left(2 S_{1}+\pi g^{\prime}(0)\right) \xi_{R}=2-\mathrm{i} \int_{-\infty}^{\infty} \hat{h}(k) \chi_{\left[-\xi_{R}, 0\right]}(-k) \mathrm{d} k
\end{aligned}
$$

where

$$
\hat{\chi}_{[a, b]}(k):=\frac{\mathrm{i}}{k}\left(\mathrm{e}^{-\mathrm{i} b k}-\mathrm{e}^{-\mathrm{i} a k}\right)
$$

is the Fourier transform of the indicator function $\chi_{[a, b]}$. Similarly, (4.8) may be expressed in the form

$$
\begin{array}{r}
\frac{2 \pi^{2}}{(1-\zeta) \tau} k \hat{h}(k)-4+2 S_{1} \hat{\chi}_{\left[-\xi_{R}, \xi_{L}\right]}(k)+\frac{2(1-\zeta) \tau}{\pi} \\
\left(\hat{\chi}_{\left[0, \xi_{L}\right]}(k)-\hat{\chi}_{\left[-\xi_{R}, 0\right]}(k)\right) \\
=-\mathrm{i}\left(\hat{h} \star \hat{\chi}_{\left[-\xi_{R}, \xi_{L}\right]}\right)(k)
\end{array}
$$

where $\star$ denotes the convolution of two functions. 
Now we invert the Fourier transforms, resulting in

$$
\begin{aligned}
\frac{\pi}{(1-\zeta) \tau} \dot{h}(t)-\chi_{\left[-\xi_{R}, \xi_{L}\right]}(t) h(t)=\frac{2 \mathrm{i}}{\pi} & \delta(t)-\frac{\mathrm{i} S_{1}}{\pi} \chi_{\left[-\xi_{R}, \xi_{L}\right]}(t) \\
& +\frac{\mathrm{i}(1-\zeta) \tau}{\pi^{2}}\left(\chi_{\left[-\xi_{R}, 0\right]}(t)-\chi_{\left[0, \xi_{L}\right]}(t)\right),
\end{aligned}
$$

while (4.17) for $\xi_{L}$ and $\xi_{R}$ may be written in terms of $h(t)$ in the form

$$
\begin{aligned}
& \left(S_{1}+\frac{(1-\zeta) \tau}{\pi}\right) \xi_{L}=1-\pi \mathrm{i} \int_{0}^{\xi_{L}} h(t) \mathrm{d} t \\
& \left(S_{1}-\frac{(1-\zeta) \tau}{\pi}\right) \xi_{R}=1-\pi \mathrm{i} \int_{-\xi_{R}}^{0} h(t) \mathrm{d} t .
\end{aligned}
$$

By integrating (4.20) with respect to $t$, we deduce from (4.21) the relations

$$
h\left(0_{ \pm}\right)= \pm \frac{\mathrm{i}(1-\zeta) \tau}{\pi^{2}}
$$

which agree with the jump conditions implied by the delta function in (4.20).

The solution of the ordinary differential equation (4.20) for $h(t)$, subject to $h(t) \rightarrow 0$ as $t \rightarrow \pm \infty$, is

$$
\begin{array}{r}
h(t)=\frac{\mathrm{i}}{\pi}\left(S_{1}-\frac{(1-\zeta) \tau}{\pi}\right)\left(1-\mathrm{e}^{(1-\zeta)\left(t+\xi_{R}\right) \tau / \pi}\right) \chi_{\left[-\xi_{R}, 0\right]}(t) \\
+\frac{\mathrm{i}}{\pi}\left(S_{1}+\frac{(1-\zeta) \tau}{\pi}\right)\left(1-\mathrm{e}^{(1-\zeta)\left(t-\xi_{L}\right) \tau / \pi}\right) \chi_{\left[0, \xi_{L}\right]}(t) .
\end{array}
$$

By substituting into the conditions (4.22) we obtain two equations for $\xi_{L}$ and $\xi_{R}$, namely,

$$
\xi_{L}=\frac{\pi}{(1-\zeta) \tau} \log \left(1+\frac{(1-\zeta) \tau}{\pi S_{1}}\right), \quad \xi_{R}=-\frac{\pi}{(1-\zeta) \tau} \log \left(1-\frac{(1-\zeta) \tau}{\pi S_{1}}\right) .
$$

Finally, by taking the Fourier transform of (4.23), we obtain the solution for $\hat{h}(k)$, and substitution back into (4.15) gives

$$
g(k)=\frac{\mathrm{i}(1-\zeta) \tau}{\pi^{2}} \frac{k}{k+\mathrm{i}(1-\zeta) \tau / \pi}\left[\left(S_{1}-\frac{(1-\zeta) \tau}{\pi}\right) \mathrm{e}^{\mathrm{i} \xi_{R} k}-\left(S_{1}+\frac{(1-\zeta) \tau}{\pi}\right) \mathrm{e}^{-\mathrm{i} \xi_{L} k}\right] .
$$

In summary, we now have explicit asymptotic solutions (4.6) for $B_{n}$, (4.24) for $\xi_{L, R}$ and (4.25) for $g(k)$, all of which depend on the two series for $\zeta$ and $S_{1}$ defined by (4.13) and (4.14). We will next analyze how these two series behave as functions of $\tau$.

4.3. Analysis of series. First consider the behavior of $S_{1}$ in the limit $\tau \rightarrow \infty$. We write (4.14) in the form

$$
S_{1}=-\frac{4 \tau}{\pi^{2}} \operatorname{Im} \sum_{j=0}^{\infty} \frac{1}{(2 j+1)} K\left(h, \sqrt{\frac{(2 j+1) \mathrm{i} \pi}{\tau}}\right)
$$

Copyright (c) by SIAM. Unauthorized reproduction of this article is prohibited. 


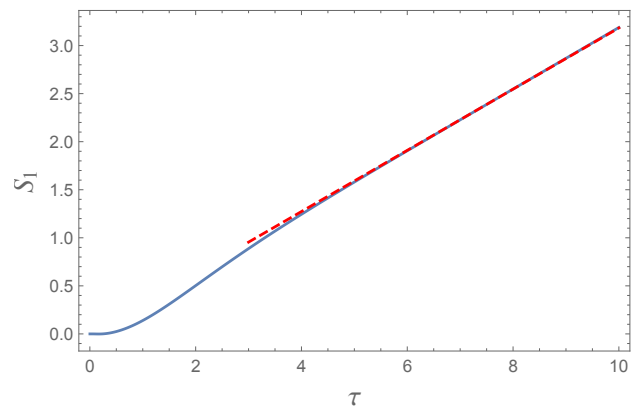

(a)

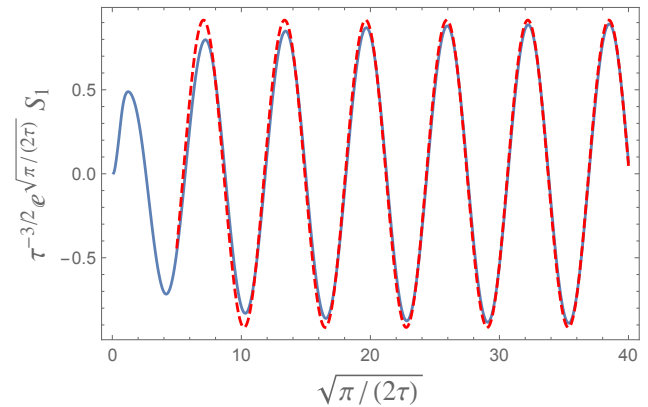

(b)

FIG. 5. (a) The series solution (4.14) for $S_{1}$ plotted versus $\tau$ with $h=2$; the red dashed line shows the large- $\tau$ asymptotic approximation (4.28). (b) The behavior near $\tau=0$ plotted using the local variable $\sqrt{\pi /(2 \tau)}$; the red dashed curve shows the approximation (4.29).

and use Euler-Maclaurin summation to get the leading-order approximation

$$
S_{1} \sim-\frac{2 \tau}{\pi^{2}} \int_{0}^{\infty} \operatorname{Im} K(h, \sqrt{\mathrm{i} s}) \frac{\mathrm{d} s}{s} .
$$

By using contour deformation, we find that the integral is equal to $-\pi / 2$ (independent of the value of $h$ ) and, therefore,

$$
S_{1} \sim \frac{\tau}{\pi} \quad \text { as } \tau \rightarrow \infty
$$

Figure 5(a) shows a plot of $S_{1}$ versus $\tau$ with $h=2$, demonstrating convergence to the limiting behavior (4.28) (plotted as a red dashed line) when $\tau$ is large.

On the other hand, in the limit $\tau \rightarrow 0$, the series in (4.14) is dominated by the first term, and we find

$$
S_{1} \sim \frac{8 h}{\pi^{5 / 2}} \tau^{3 / 2} \mathrm{e}^{-\sqrt{\pi / 2 \tau}} \sin \left(\sqrt{\frac{\pi}{2 \tau}}+\frac{\pi}{4}\right) \quad \text { as } \tau \rightarrow 0 .
$$

Convergence to this asymptotic behavior is shown in Figure 5(b) by plotting $S_{1}$, divided by the exponentially small prefactor $\tau^{3 / 2} \mathrm{e}^{-\sqrt{\pi / 2 \tau}}$, versus $\sqrt{\pi / 2 \tau}$, with the red dashed curve showing the sinusoidal behavior predicted by (4.29). We observe that $S_{1}$ becomes exponentially small while undergoing an infinity of oscillations in the limit $\tau \rightarrow 0$.

Now we perform analogous calculations on the series solution (4.13) for $\zeta$. For large $\tau$, Euler-Maclaurin summation leads to the approximation

$$
\begin{aligned}
\sum_{n=-\infty}^{\infty} \frac{1}{n^{2}} K\left(h, \sqrt{\frac{\mathrm{i} n \pi}{\tau}}\right) & =2 \operatorname{Re} \sum_{j=0}^{\infty} \frac{1}{(2 j+1)^{2}} K\left(h, \sqrt{\frac{\mathrm{i}(2 j+1) \pi}{\tau}}\right) \\
& \sim \frac{\pi^{2}}{4}+\frac{\pi}{\tau} \int_{0}^{\infty} \frac{\operatorname{Re} K(h, \sqrt{\mathrm{i} s})-1}{s^{2}} \mathrm{~d} s .
\end{aligned}
$$

By contour deformation, the integral is found to be given by $-(h+2) \pi /(4 h)$ and thus we get the approximation

$$
\zeta \sim \frac{2+h}{h \tau} \text { as } \tau \rightarrow \infty
$$

which is plotted as a dashed curve in Figure 6(a). 


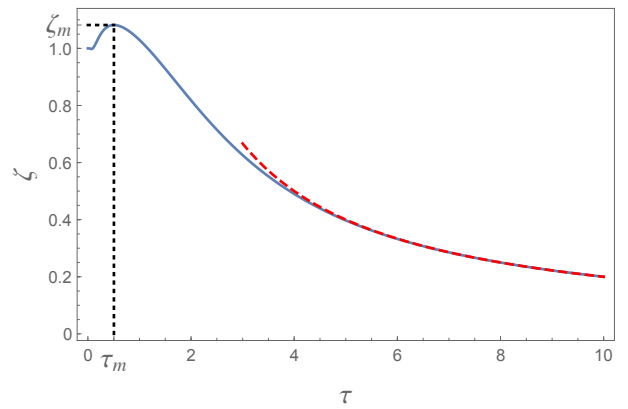

(a)

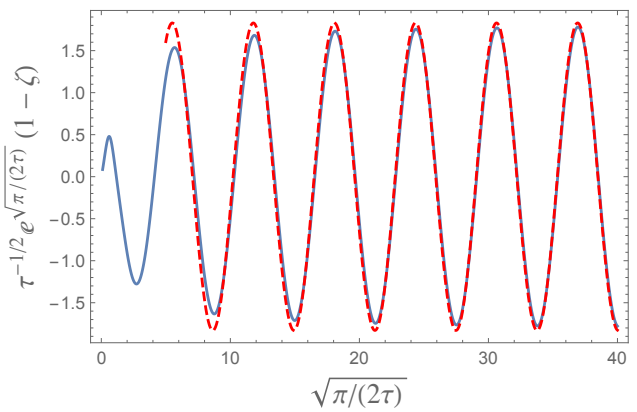

(b)

FIG. 6. (a) The series solution (4.13) for $\zeta$ plotted versus $\tau$ with $h=2$; the red dashed line shows the large- $\tau$ asymptotic approximation (4.31); the maximum value of $\zeta$ is labeled as $\zeta_{m}(h)$, corresponding to $\tau=\tau_{m}(h)$, with $\zeta_{m}(2) \approx 1.08187$ and $\tau_{m}(2) \approx 0.504702$. (b) The behavior near $\tau=0$ plotted using the local variable $\sqrt{\pi / 2 \tau}$; the red dashed curve shows the approximation (4.32).

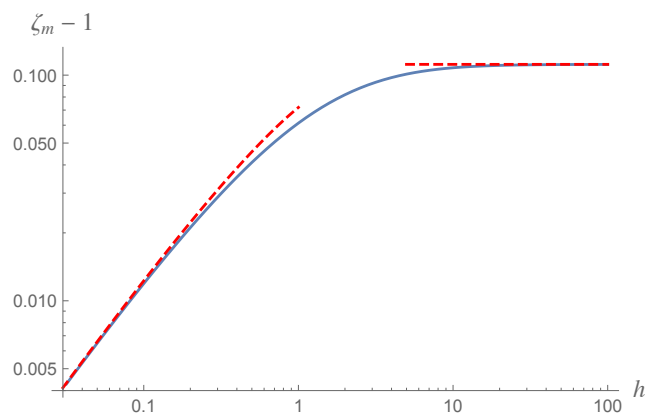

(a)

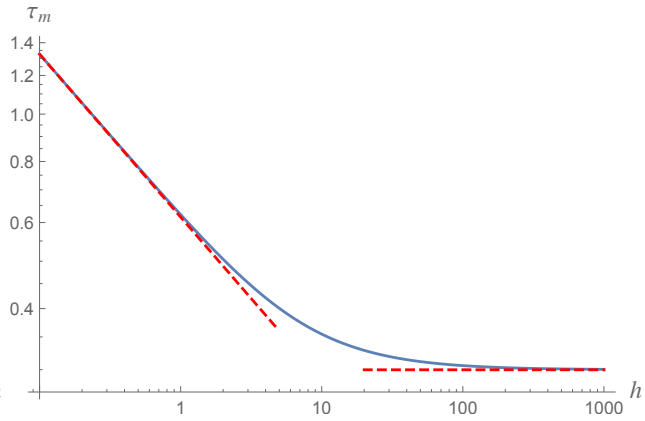

(b)

Fig. 7. (a) The maximum value $\zeta_{m}(h)$ of $\zeta$ and (b) the corresponding value $\tau_{m}(h)$ of $\tau$ plotted versus $h$. The red dashed curves show the asymptotic behaviors (4.37) as $h \rightarrow 0$ and the limiting values $\zeta_{m}(h) \rightarrow \zeta_{m}(\infty) \approx 1.11162$ and $\tau_{m}(h) \rightarrow \tau_{m}(\infty) \approx 0.299807$ as $h \rightarrow \infty$.

For small $\tau$, the series is again dominated by the first term, and we find

$$
1-\zeta \sim \frac{16 h}{\pi^{5 / 2}} \tau^{1 / 2} \mathrm{e}^{-\sqrt{\pi / 2 \tau}} \cos \left(\sqrt{\frac{\pi}{2 \tau}}+\frac{\pi}{4}\right) \quad \text { as } \tau \rightarrow 0 .
$$

In Figure 6(b), we plot $(1-\zeta)$, divided by the exponentially small prefactor $\tau^{1 / 2} \mathrm{e}^{-\sqrt{\pi / 2 \tau}}$, versus $\sqrt{\pi / 2 \tau}$, and demonstrate convergence to the sinusoidal behavior predicted by (4.32), which is shown as the dashed red curve. The value of $\zeta$ undergoes an infinity of oscillations about unity as $\tau \rightarrow 0$, and therefore $\tau$ is not uniquely determined as a function of $\zeta$ unless the range is restricted. As illustrated in Figure 6(a), we denote by $\zeta_{m}(h)$ and $\tau_{m}(h)$ the maximum value of $\zeta$ and the corresponding value of $\tau$, with $\zeta_{m}(2) \approx 1.08187$ and $\tau_{m}(2) \approx 0.504702$. Thus there is a one-to-one relation between $\zeta$ and $\tau$ provided $0<\zeta<\zeta_{m}$ and $\tau_{m}<\tau<\infty$.

In Figure 7 we show how $\zeta_{m}(h)$ and $\tau_{m}(h)$ vary as functions of the heat transfer parameter $h$. As $h \rightarrow \infty$, they both approach constant values $\zeta_{m}(\infty) \approx 1.11162$ and $\tau_{m}(\infty) \approx 0.299807$, which are determined numerically by maximizing the series 
solution (4.13) as a function of $\tau$ with $K(\infty, s)=\operatorname{sech} s$. For future reference, the corresponding value of $S_{1}$ is found to be $\approx 0.0170272$.

The behavior as $h \rightarrow 0$ is more subtle. We note from (4.31) that the asymptotic limit of $\zeta$ as $\tau \rightarrow \infty$ appears to be confounded if $h$ is also asymptotically small. Indeed, we find that a distinguished limit occurs when $\tau=O\left(h^{-1 / 3}\right)$ as $h \rightarrow 0$. We therefore make the substitution

$$
\tau=h^{-1 / 3} \tilde{\tau}
$$

before expanding (4.13) in the limit of small $h$, resulting in

$$
\zeta-1 \sim \frac{h}{6}-h^{4 / 3}\left(\frac{\tilde{\tau}^{2}}{12}+\frac{I_{1}}{\tilde{\tau}}\right)+\cdots,
$$

where

$$
I_{1}=\frac{4}{\pi} \int_{0}^{\infty}\left(\frac{1}{6 s^{2}}-\operatorname{Re}\left[\frac{\operatorname{cosech}(\sqrt{\mathrm{i} s})}{(\mathrm{i} s)^{5 / 2}}\right]\right) \mathrm{d} s .
$$

By contour deformation, we find that $I_{1}=7 / 180$ and therefore

$$
\zeta-1 \sim \frac{h}{6}-\frac{h^{4 / 3}}{12}\left(\tilde{\tau}^{2}+\frac{7}{15 \tilde{\tau}}\right)+\cdots .
$$

It follows that $\zeta$ is maximized when $\tilde{\tau}=(7 / 30)^{1 / 3}$ and hence

$$
\zeta_{m}(h) \sim 1+\frac{h}{6}-\left(\frac{7}{30}\right)^{2 / 3} \frac{h^{4 / 3}}{4}, \quad \tau_{m}(h) \sim\left(\frac{7}{30 h}\right)^{1 / 3} \quad \text { as } h \rightarrow 0 .
$$

These asymptotic limits are plotted as red dashed curves in Figure 7.

4.4. Behavior of solutions. Figure 6 a shows the leading-order relation (4.13) between $\zeta$ and $\tau$ obtained in the limit $\alpha \rightarrow \infty$. In practice, the value of the parameter $\zeta$ is to be specified and the half-period $\tau$ of the periodic solution (if one exists) is to be determined. In Figure 8(a), we therefore plot the same relation (4.13) with the axes swapped to show $\tau$ as a function of $\zeta$, here with $h=2$. Large-amplitude oscillatory solutions exist only if $\zeta$ is less than the critical value $\zeta_{m}(2) \approx 1.082$. The uppermost solution branch with $\tau>\tau_{m}(2) \approx 0.5047$ is presumed to be stable, as will be verified below by comparison with numerical solutions. Since the curve in Figure 8(a) undergoes an infinity of oscillations about $\zeta=1$ as $\tau \rightarrow 0$, there is an infinite set of solution branches in $\tau<\tau_{m}$ clustered around $\zeta=1$. One might conjecture that these branches are alternately stable and unstable but, even if this were true, the additional stable branches would exist only for a tiny range of values of $\zeta$ and would be attained for a negligible set of initial conditions. We therefore consider only the top solution branch represented by the solid curve in Figure 8(a) to be physically relevant.

On the other hand the trivial solution with $H(t) \equiv 0$ (and therefore zero period) exists for all values of $\zeta$, and we know from Figure 3 that it is stable if $\zeta$ exceeds a critical value $\zeta_{*}(h) \approx 1.054$ when $h=2$. It follows that there is a narrow range of values of $\zeta$ for which both the steady solution and the oscillatory solution are 


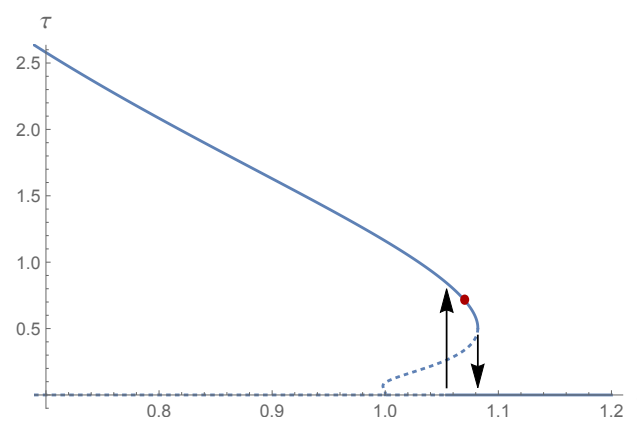

(a)

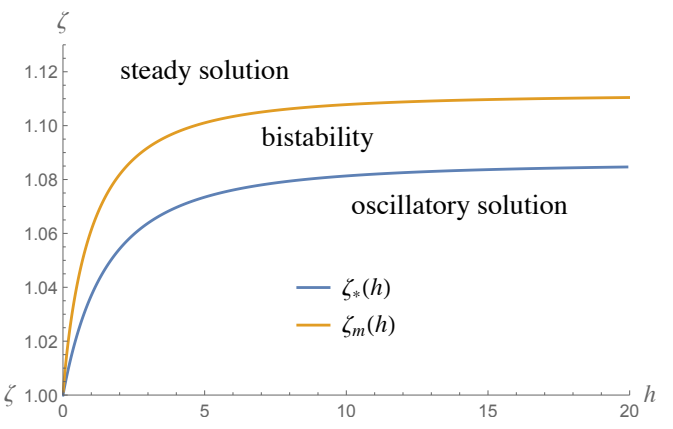

(b)

FIG. 8. (a) The relation (4.13) between the period $\tau$ and the parameter $\zeta$ with $h=2$. The top branch is plotted as a solid curve, and the lower branches, presumed to be physically irrelevant, as dotted curves. Also shown is the steady solution $\tau=0$, which is stable for $\zeta \gtrsim \zeta_{*}(2) \approx 1.054$. The red dot shows the period $\tau \approx 0.715$ when $\zeta=1.07$ determined numerically from the computation shown in Figure $9(\mathrm{~b})$. (b) The $(h, \zeta)$ parameter space. The blue curve shows the minimum value $\zeta_{*}(h)$ for stability of the steady solution; the orange curve shows the maximum value $\zeta_{m}(h)$ for existence of the large-amplitude oscillatory solution. Between the two curves, both solutions are possible.

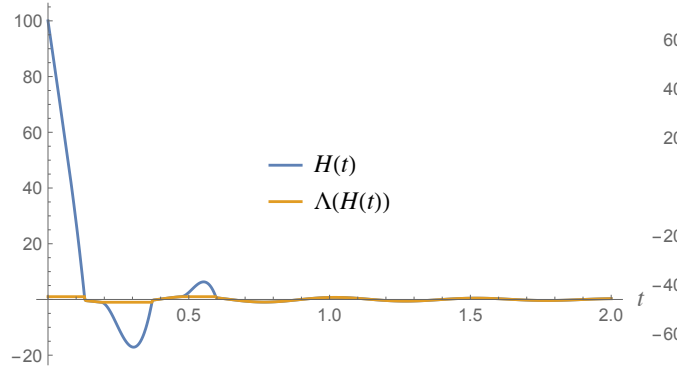

(a) $H(0)=100$.

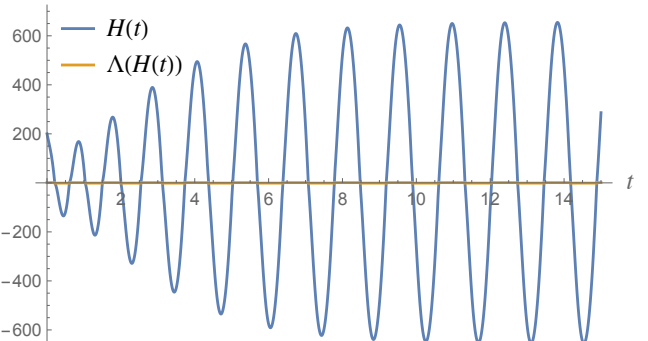

(b) $H(0)=200$.

FIG. 9. Numerical solutions of (2.13), with parameter values $h=2, \alpha=10^{4}, \eta=1.07 \times 10^{4}$ and two different initial conditions.

attractors for the system. Moreover, if the value of the parameter $\zeta$ were to be gradually varied, we would expect the system to exhibit hysteresis, as indicated by the arrows in Figure 8(a).

The general pattern illustrated in Figure 8(a) is repeated for other values of $h$. As shown in Figure 8(b), there is always a critical value $\zeta_{m}$ of $\zeta$ above which the oscillatory solution ceases to exist, and a critical value $\zeta_{*}$ of $\zeta$ below which the steady solution loses stability. In the region between these two curves, the system is bistable, and which of the two behaviors emerges will depend on the initial conditions.

We illustrate the possible behavior in Figure 9, where we show numerical solutions of (2.13) with $h=2, \alpha=10^{4}$, and $\zeta=1.07$, which is in the predicted bistable region. We use initial conditions (a) $H(0)=100$, (b) $H(0)=200$. In the former case, the system undergoes a small number of nonlinear oscillations of decreasing amplitude, before rapidly decaying towards the zero solution. In the latter case, the initial perturbation of the system is large enough to push it into the large-amplitude oscillatory state. Furthermore, we read off the period $\tau \approx 0.715$ of the numerical 


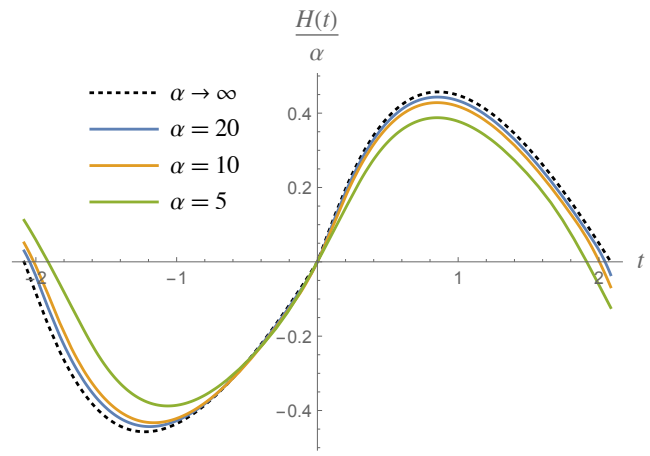

(a)

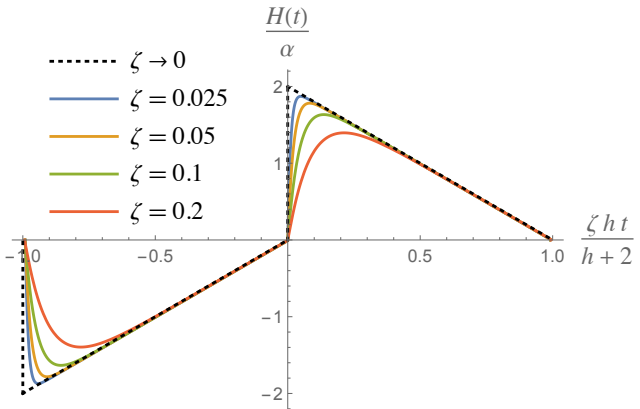

(b)

FIG. 10. (a) Normalized periodic solutions for $H(t) / \alpha$ with $h=2, \zeta=\eta / \alpha=0.8$, and three different values of $\alpha$. The limiting solution (4.38) as $\alpha \rightarrow \infty$ is plotted as a black dotted curve. (b) Normalized periodic solutions for $H(t) / \alpha$ plotted versus normalized time $\zeta h t /(h+2)$ with $h=2$, $\alpha=100$, and varying values of $\zeta$. The limiting solution (4.39) as $\zeta \rightarrow 0$ is plotted as a black dotted curve.

solution and plot this computed value as a red dot in Figure 8(a), where we see that it appears to lie precisely on the $(\tau, \zeta)$ curve predicted by the asymptotic theory.

For values of $\zeta$ where oscillatory solutions exist, we recover $H(t)$ from the Fourier series (3.1), which to leading order in $\alpha$ reduces to

$$
H(t) \sim-\frac{2 \alpha \tau}{\pi^{2}} \sum_{n=-\infty}^{\infty}\left(\frac{1-\zeta-K_{n}}{n^{2}}\right) \mathrm{e}^{\mathrm{i} n \pi t / \tau}
$$

In Figure 10(a), we show periodic solutions for $H(t)$, normalized by dividing by $\alpha$, found by solving (2.13) numerically. We keep $h=2$ and $\zeta=0.8$ fixed while varying the value of $\alpha$. As the value of $\alpha$ increases, the curves indeed collapse onto the predicted limiting solution (4.38).

In Figure 10(b) we show computed periodic solutions for $H(t)$, again normalized by dividing by $\alpha$ and now with time also normalized by the predicted period $\tau \sim$ $(h+2) / h \zeta$. We fix $h=2$ and a large value of $\alpha=100$, and then vary the value of $\zeta$. As $\zeta$ decreases towards zero, the behavior of $H(t)$ becomes increasingly asymmetric, with the pins rapidly moving away from each other before more slowly coming back together. Although the period tends to infinity as $\zeta \rightarrow 0$, the amplitude of the solution remains finite, approaching the limiting sawtooth profile, plotted as a black dotted curve in Figure 10(b), with

$$
\frac{H(t)}{\alpha} \sim \frac{2+h}{h}\left(1-\frac{t}{\tau}\right) \quad \text { for } 0<t<\tau .
$$

The leading-order normalized amplitude of the solution is given by

$$
\frac{\|H\|}{\alpha}=\sqrt{\frac{1}{\tau} \int_{0}^{\tau} H(t)^{2} \mathrm{~d} t} \sim \sqrt{\sum_{n=-\infty}^{\infty}\left|B_{n}\right|^{2}}=: A
$$

with $B_{n}$ given by (4.6). In Figure 11(a), we plot the amplitude $A$ versus $\zeta$ with $h=2$. The limit $A \rightarrow(2+h) / h \sqrt{3}$ as $\zeta \rightarrow 0$ is inferred from the large- $\tau$ behavior 


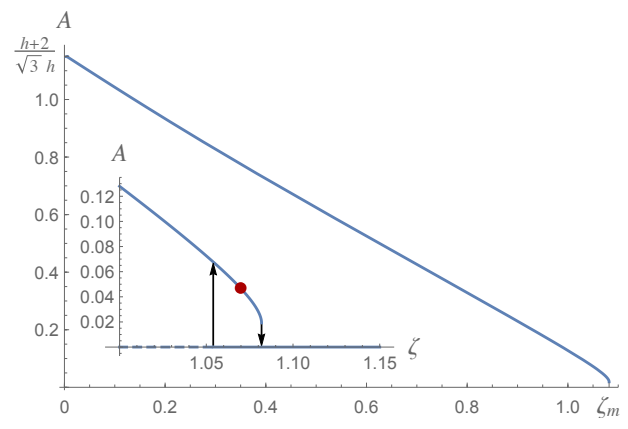

(a)

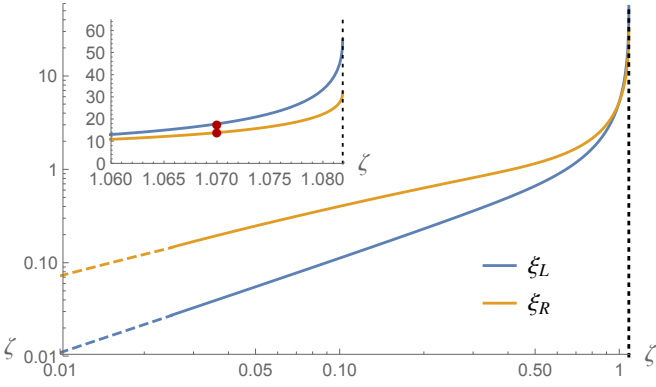

(b)

FIG. 11. (a) The normalized amplitude $A=\|H\| / \alpha$, given by (4.40), plotted versus $\zeta$ with $h=2$. The inset shows a neighborhood of $\zeta=\zeta_{m}(2) \approx 1.08187$, where the nonzero solution branch terminates with min $A \approx 0.019123$, while the zero solution is unstable for $\zeta<\zeta_{*}(2) \approx 1.054$. The arrows indicate the possibility of hysteresis as $\zeta$ varies; the red dot shows the amplitude computed from the simulation showed in Figure 9(b). (b) The normalized positions $\xi_{L}$ and $\xi_{R}$ of the free boundaries, given by (4.24), plotted versus $\zeta$ with $h=2$. The dashed curves show the asymptotic behaviors (4.41); the black dotted line shows the maximum value $\zeta_{m}(2) \approx 1.08187$. The inset shows the behavior near $\zeta=\zeta_{m}$ with the red dots showing the values of $\xi_{L}$ and $\xi_{R}$ computed from the simulation shown in Figure 9(b).

(4.31). As in Figure 8(a), we observe that the nonzero solution branch terminates with $A \approx 0.019123$ at the finite value $\zeta=\zeta_{m}(2) \approx 1.054$ and, again, we see the possibility of hysteresis if $\zeta$ is varied. The red dot in Figure 11(a) shows the value of $A$ determined from the simulation shown in Figure 9(b) by numerically integrating over a period, and we see that the agreement with the asymptotic prediction is excellent.

The normalized positions of the free boundaries $\xi_{L}$ and $\xi_{R}$, where each pin gains or loses contact with the wheel, are given by (4.24). With $S_{1}$ and $\zeta$ given by (4.13) and (4.14), we can thus parametrically plot $\xi_{L}$ and $\xi_{R}$ versus $\zeta$, as shown in Figure 11(b) with $h=2$. The behavior as $\zeta \rightarrow 0$ may be inferred from the behaviors (4.28) and (4.31) of $S_{1}$ and $\zeta$ as $\tau \rightarrow \infty$, viz,

$$
\xi_{L} \sim\left(\frac{\pi h \log 2}{h+2}\right) \zeta, \quad \xi_{L} \sim\left(\frac{\pi h}{h+2}\right) \zeta \log \left(\frac{1}{\zeta}\right) \quad \text { as } \zeta \rightarrow 0,
$$

which are shown as dashed curves in Figure 11(b). As $\zeta$ increases, both $\xi_{L}$ and $\xi_{R}$ increase, but the curves terminate at large but finite values as $\zeta$ approaches its maximum value $\zeta_{m} \approx 1.08187$ when $h=2$, as demonstrated by the inset in Figure 11(b). The red dots show the values of $\xi_{L}$ and $\xi_{R}$ computed by finding the values of $t$ where $H(t)$ crosses the values $-1,0$, and 1 in the simulation shown in Figure 9(b). Evidently the asymptotic results accurately predict the computed values.

In summary, as the parameter $\zeta$ decreases towards zero, the normalized amplitude $A$ of the oscillations approaches a finite value, but the period $\tau$ of the oscillations tends to infinity. At the same time, the values of $\xi_{L}$ and $\xi_{R}$ both tend to zero, implying that just one pin is in contact virtually all of the time.

On the other hand, it is clear in Figures 8 and 11 that the nonzero solution branch terminates with finite, nonzero values of $\tau, A, \xi_{L}$, and $\xi_{R}$, implying that there is an abrupt transition to the zero solution as $\zeta$ increases through the critical value $\zeta_{m}$. In Figure 12 we demonstrate that the same observation is true for all values of the heat transfer parameter $h$. In Figure 12(a), we plot the minimum value of the amplitude $A$, 


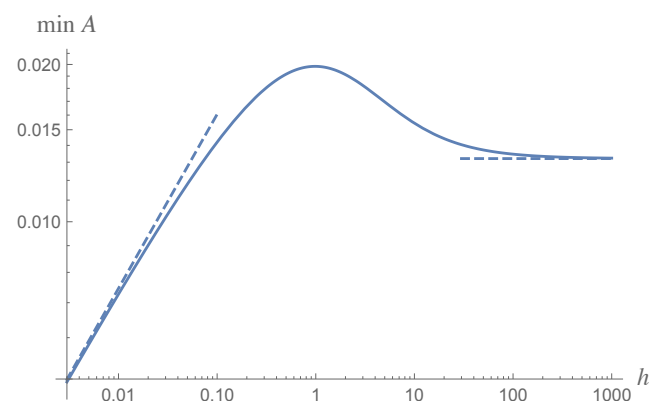

(a)

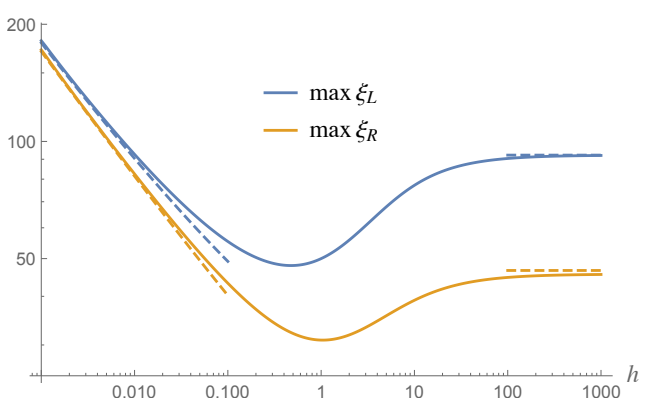

(b)

FIG. 12. (a) The minimum value of the amplitude $A$, attained at $\zeta=\zeta_{m}(h)$, plotted versus $h$. The dashed curves shows the asymptotic behavior (4.43) and the limiting value $A \rightarrow 0.0132095$ as $h \rightarrow \infty$. (b) The maximal values of $\xi_{L}$ and $\xi_{R}$, corresponding to $\zeta=\zeta_{m}(h)$, plotted versus $h$. The dashed curves show the asymptotic behavior (4.43) and the limiting values $\xi_{L} \rightarrow 92.2247$ and $\xi_{R} \rightarrow 46.6132$ as $h \rightarrow \infty$.

corresponding to $\zeta=\zeta_{m}(h)$, versus $h$, and in Figure 12(b), we plot the corresponding maximal values of $\xi_{L}$ and $\xi_{R}$ versus $h$. Counterintuitively, the behavior of all three functions is nonmonotonic. As $h \rightarrow \infty$, we see that $\min A, \max \xi_{L}$, and $\max \xi_{R}$ all approach constant values determined by substituting the limiting values of $\zeta_{m}$ and $\tau_{m}$ from Figure 7 into (4.24) and (4.40).

A little more work is required to understand the behavior as $h \rightarrow 0$. We substitute the asymptotic behavior (4.37) of $\tau_{m}$ as $h \rightarrow 0$ into the formula (4.14) for $S_{1}$ and expand to get

$$
S_{1} \sim \frac{\tilde{\tau}^{2} h^{1 / 3}}{2 \pi}-\frac{\tilde{\tau} h^{2 / 3}}{6 \pi}+\cdots \quad \text { when } \tau=h^{-1 / 3} \tilde{\tau} \text { and } h \rightarrow 0
$$

where we recall that the extremal occurs when $\tilde{\tau}=(7 / 30)^{1 / 3}$. Substituting (4.37) and (4.42) into (4.24) and (4.40), we obtain the asymptotic behaviors of $A, \xi_{L}$, and $\xi_{R}$, namely,

$$
A \sim \frac{7^{2 / 3} h^{1 / 3}}{2 \cdot 30^{7 / 6}}, \quad \xi_{L} \sim\left(\frac{30}{7}\right)^{2 / 3} \frac{2 \pi}{h^{1 / 3}}+\frac{30 \pi}{7}, \quad \xi_{R} \sim\left(\frac{30}{7}\right)^{2 / 3} \frac{2 \pi}{h^{1 / 3}}+\frac{10 \pi}{7}
$$

as $h \rightarrow 0$, which are plotted using dashed curves in Figure 12 .

Finally, in Figure 13 we show the results of a simulation in which $\zeta$ is varied as a function of $t$ according to

$$
\zeta(t)-1= \begin{cases}0.15\left(\frac{t}{50}-1\right), & 50<t<100 \\ -0.15\left(\frac{t}{50}-3\right), & 100<t<150 \\ 0, & \text { otherwise }\end{cases}
$$

so that it ramps up and down through the bifurcation points $\zeta_{m}$ and $\zeta_{*}$. The blue curve in Figure 13 shows rapid nonlinear oscillations in $H(t)$, and the red dots show instantaneous values of $\|H\|$ computed by integrating $H(t)$ across successive halfperiods. The green curve in Figure 13 shows how $\zeta-1$ varies with $t$, and the yellow curve shows the predicted equilibrium amplitude $A(\zeta)$ as plotted in Figure 11(a). 


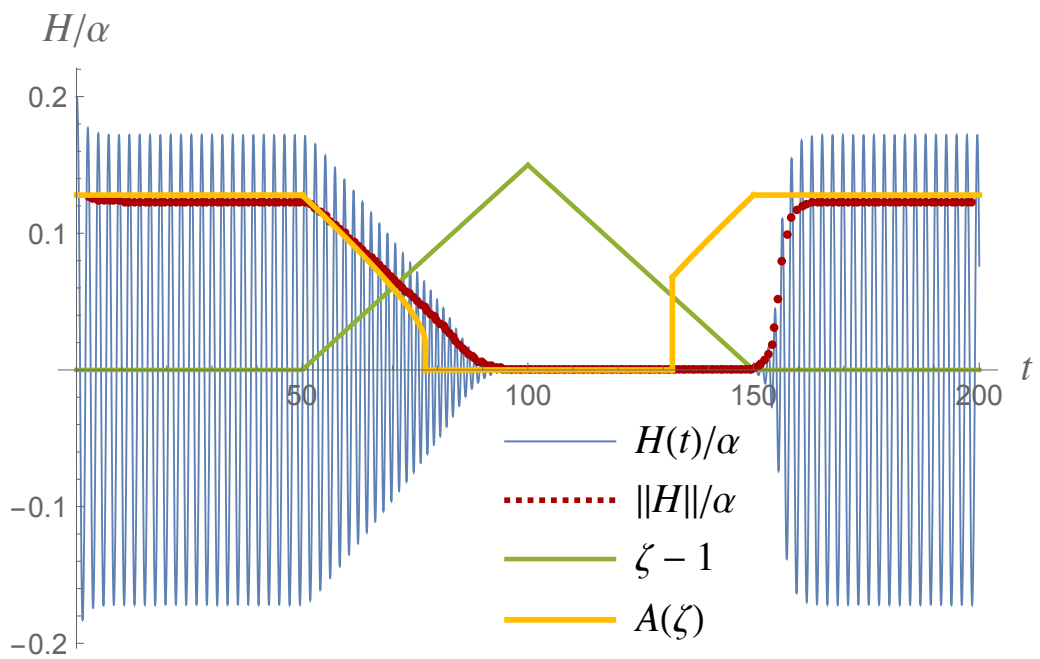

FIG. 13. The blue curve shows results of a simulation run with $h=2, \alpha=20$, and $\eta / \alpha=\zeta(t)$ with $\zeta(t)-1$ given by (4.44) and plotted as a green curve. The red dots show the amplitude $\|H\|$ computed by integrating the blue curve over successive half-periods. The yellow curve shows the equilibrium amplitude for each value of $\zeta$, found by following Figure 11(a) in the direction of the arrows.

Initially $\zeta=1$, and the computed amplitude closely follows the asymptotic prediction. The predicted amplitude $A$ decreases as $\zeta$ increases, until $\zeta$ reaches the fold bifurcation value $\zeta_{m}$ and the equilibrium $A$ jumps to zero. At this point, the computed amplitude lags behind the equilibrium curve due to rate effects, but it ultimately does converge to the zero solution. As $\zeta$ later decreases through the Hopf bifurcation value $\zeta_{*}$, the equilibrium amplitude jumps to the nonlinear branch, but the computed amplitude displays a classic delayed bifurcation caused by the slow transition of $\zeta$ through the critical value.

5. Conclusions. The dynamical system (2.13) studied in this paper possesses two distinctive features, namely, nonsmoothness, through the function $\Lambda(H)$, and nonlocality, through the history-dependent functional $Q[\Lambda]$. We find that, generically, the system converges either to the trivial state $H=0$ or to a state characterized by highly nonlinear oscillations, with $\Lambda$ repeatedly switching between its extreme values of \pm 1 . Since $\Lambda(H) \equiv H$ when $|H|<1$, the problem is precisely linear, and consequently the behavior of $H(t)$ is purely exponential, for $|H|<1$. The solution must therefore either decay to zero or grow until $|H|=1$ and nonlinear effects finally kick in. As a consequence of the degenerate nonlinearity in the model, there can exist no nontrivial oscillatory solutions with amplitude less than 1 , other than for exceptional parameter values that lie precisely on the stability boundary,

Our use of the quantity $H$, to unfold the discontinuous contact relation between the force and displacement, is reminiscent of the use of enthalpy in a Stefan problem (see [5], for example). In a prototypical Stefan problem, a substance can exist either in one of two phases (solid and liquid, say), or as a mixture of the two phases known as a mushy region. Similarly, our system has two "pure" phases, with either pin 1 or pin 2 in contact, and a "mixed" phase, where they share the load. In a simple Stefan problem, the liquid fraction is a discontinuous set-valued function of temperature, similar to Figure 1(a), but a continuous function of the enthalpy, as in Figure 1(b). 
Finally, the enthalpy satisfies a single PDE to be solved everywhere, with the locations of any phase boundaries determined a posteriori. Similarly, our formulation results in a single differential equation (2.13) for the enthalpy-like quantity $H$ and removes the need to check at each time which phase of the motion we are in.

Our model system (2.13) is based on a number of simplifying modeling assumptions, which could be relaxed to include more physics. For example, we describe the heat flow in each pin using the one-dimensional heat equation, which could be generalized to include heat loss to the surrounding atmosphere. Similarly, we model the base of each pin as being fixed and at constant temperature, and one could instead include mechanical and thermal coupling between the pins in the boundary conditions at $X=0$. Finally, one can incorporate heat transfer between each pin and the moving surface in the boundary condition (2.1c). If one then allows the corresponding heat transfer coefficient to depend on the contact force $F_{j}$, then the uniform state may be unstable even when there is only the $N=1$ pin (see, e.g., $[1,7]$ ).

Our analysis proceeds by first using Fourier decomposition for an assumed periodic solution, and then taking the limit $\alpha \rightarrow \infty$ in the resulting system of linear and nonlinear algebraic equations. In principle, one could alternatively attempt to analyze the PDE problem (2.12) directly but, in practice, such an approach is complicated by the multiple time scales in the problem. The rapid switches between $\Lambda= \pm 1$ occur over a short time scale of order $1 / \alpha$, but are subject to a background temperature field that evolves over an $O(1)$ time scale. Our Fourier decomposition assists the asymptotic analysis by effectively separating the fast and slow modes.

The utility of our approach rests on the following general properties of the system (2.13). Although $\Lambda$ is given as a function of $H$ by (2.11), we treat them as independent functions of $t$, which are then related by a linear integro-differential equation (2.13). Moreover, we can view $\Lambda(t)$ is the product of $H(t)$ with a gate function, parametrized by the period $\tau$ of the oscillation and the times $t_{\mathrm{L}}$ and $t_{\mathrm{R}}$ at which $H(t)$ passes through the critical values \pm 1 . Given the values of these three parameters, the problem is therefore entirely linear, and thus solvable in principle using linear methods. It then only remains to solve three nonlinear algebraic relations for $\left\{\tau, t_{\mathrm{L}}, t_{\mathrm{R}}\right\}$.

Our analysis reveals several features of the dynamics that are not obvious a priori. As the parameter $\zeta$ decreases, the oscillatory solution branch appears abruptly with large $O(\alpha)$ amplitude, rather than growing smoothly out of the trivial solution. There is always a small but finite range of values of $\zeta$ for which both oscillatory and steady solutions are stable; in such a parameter regime, the initial conditions determine which attractor the system adopts, and there is the possibility of hysteresis between the two branches if $\zeta$ is gradually varied. As $\zeta$ is decreased towards zero, the trivial solution ceases to be stable, and the oscillations become increasingly asymmetric.

The generalization of our model to $N>2$ pins is considered in [11]. An enthalpylike formulation analogous to (2.10) allows the problem to be cast as a nonsmooth, history-dependent dynamical system on $\mathbb{R}^{N-1}$. Numerical simulations for $N=3$ indicate that, when $\alpha$ is large, the system again spends most of the time with just one pin in contact, and the load transfers between pins occur very rapidly. However, the increase in dimensionality leads to an increase in the variety of possible solutions; in particular, the system can produce apparently chaotic behavior. It is hoped that an asymptotic approach similar to the one used here will allow the behavior to be characterized and quantified for larger values of $N$ and, indeed, for other dynamical systems of similar type. 


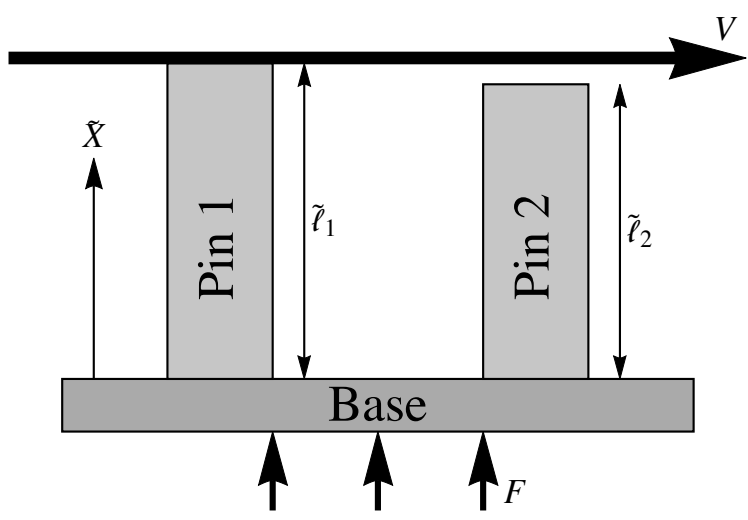

FIG. 14. A sketch of the two-pin problem. Each pin is clamped to the base at $\tilde{X}=0$, and the whole assembly is pressed with force $F$ against a surface moving tangentially at speed $V$. The length of pin $j$ is labeled $\tilde{\ell}_{j}$; here $\tilde{\ell}_{1}>\tilde{\ell}_{2}$ so pin 1 is in contact with the moving surface but pin 2 is not.

\section{Appendix A. Derivation of the model.}

Here we give a brief derivation of the governing equations (2.1)-(2.3). Figure 14 shows an illustrative sketch for the case of $N=2$ pins.

The dimensional temperature $\tilde{T}_{j}$ in pin $j=1, \ldots, N$ satisfies the heat equation

$$
\kappa \frac{\partial^{2} \tilde{T}_{j}}{\partial \tilde{X}^{2}}=\frac{\partial \tilde{T}_{j}}{\partial \tilde{t}}
$$

where $\kappa$ is the thermal diffusivity (assumed constant), $\tilde{X}$ is the Lagrangian distance from the clamped base, and $\tilde{t}$ is time. At the clamped end, we impose Newton's law of cooling, that is,

$$
k \frac{\partial \tilde{T}_{j}}{\partial \tilde{X}}=h_{\mathrm{A}}\left(\tilde{T}_{j}-T_{\mathrm{a}}\right) \quad \text { at } X=0,
$$

where $k$ is the thermal conductivity, $h_{\mathrm{A}}$ is a heat transfer coefficient, and $T_{\mathrm{a}}$ is the ambient temperature. The other end of each pin is pressed with force $\tilde{F}_{j}$ against a surface moving tangentially at constant speed $V$, resulting in a frictional heat flux given by

$$
k \frac{\partial \tilde{T}_{j}}{\partial \tilde{X}}=\mu h_{\mathrm{W}} V \tilde{F}_{j} \quad \text { at } \tilde{X}=\tilde{L}_{j},
$$

where $\mu$ is the coefficient of friction, and $h_{\mathrm{W}}$ is a second heat transfer coefficient. The boundary condition (A.3) effectively treats the sliding surface as an insulating boundary; this assumption may be relaxed to include heat transfer between the pin and the sliding surface as in $[1,7]$.

According to Archard's law [4], each pin suffers frictional wear at a rate proportional to $\tilde{F}_{j} V$ and, hence, the material length $\tilde{L}_{j}(\tilde{t})$ satisfies

$$
\frac{\mathrm{d} \tilde{L}_{j}}{\mathrm{~d} \tilde{t}}=-\beta \mu V \tilde{F}_{j},
$$

where $\beta$ is a material constant of proportionality. The corresponding Eulerian length $\tilde{\ell}_{j}$ is found by incorporating the effects of elastic compression and thermal expansion, resulting in 


$$
\tilde{\ell}_{j}=\tilde{L}_{j}\left(1-\frac{\tilde{F}_{j}}{E A}\right)+\frac{\alpha_{\mathrm{T}}}{3} \int_{0}^{\tilde{L}_{j}} \tilde{T}_{j} \mathrm{~d} \tilde{X}
$$

where $E, A$, and $\alpha_{\mathrm{T}}$ are the Youngs modulus, cross-sectional area, and thermal expansion coefficient of each pin.

Finally, we impose a contact condition with the moving surface, whose distance from the base must equal the length, $M$ say, of the longest pin. If pin $j$ makes contact with the surface, then $\tilde{\ell}_{j}=M$ and $\tilde{F}_{j} \geq 0$, while if does not, then $\tilde{\ell}_{j}<M$ and $\tilde{F}_{j}=0$. Assuming that the net normal force $F$ applied to the whole assembly is specified and constant, we deduce the complementarity conditions

$$
\begin{aligned}
\tilde{\ell}_{j} \leq M, \quad \tilde{F}_{j} \geq 0, \quad & \left(M-\tilde{\ell}_{j}\right) \tilde{F}_{j}=0 \quad(j=1, \cdots, N) \\
& \text { and } \quad \sum_{j} \tilde{F}_{j}=F .
\end{aligned}
$$

We nondimensionalize as in [13], defining

$$
\left(\tilde{X}, \tilde{L}_{j}, \tilde{\ell}_{j}\right)=L_{0}\left(X, L_{j}, \ell_{j}\right), \quad \tilde{t}=\frac{L_{0}^{2}}{\kappa} t, \quad \tilde{T}_{j}=T_{\mathrm{a}}+\frac{h_{\mathrm{W}} \mu V F L_{0}}{k} T_{j}, \quad \tilde{F}_{j}=F F_{j},
$$

where $L_{0}$ is a typical initial length for each pin. The model contains four dimensionless parameters

$$
\delta=\frac{F}{E A}, \quad \alpha=\frac{E A \alpha_{\mathrm{T}}}{3} \frac{h_{\mathrm{W}} \mu V L_{0}}{k}, \quad \eta=\frac{\beta \mu V L_{0} E A}{\kappa}, \quad h=\frac{h_{\mathrm{A}} L_{0}}{k},
$$

which measure the typical elastic strain, thermal expansion, wear rate, and heat transfer with the arm, respectively. For typical experiments with metal pins, the relative compression $\delta$ is extremely small, and we will therefore take the limit $\delta \rightarrow 0$.

The dimensionless forms of the governing equations (A.4) for the Lagrangian and Eulerian lengths of pin $j$ are written

$$
\frac{\mathrm{d} L_{j}}{\mathrm{~d} t}=-\delta \eta F_{j}, \quad \ell_{j}=L_{j}\left(1-\delta F_{j}\right)+\delta \alpha \int_{0}^{L_{j}} T_{j} \mathrm{~d} X .
$$

It follows that, over $O(1)$ time scales, both $L_{j}$ and $\ell_{j}$ remain close to their initial values, which we take to be 1 by our normalization, and we can therefore write

$$
L_{j}=1-\delta D_{j}, \quad \ell_{j}=1+\delta d_{j},
$$

where $D_{j}(t)$ and $d_{j}(t)$ are $O(1)$. In the limit $\delta \rightarrow 0$, the dimensionless version of the model (A.1)-(A.5) thus reduces to (2.1)-(2.3) in the text.

Acknowledgments. The author is grateful for helpful discussions with Ademola Akande, Prof. J. R. Ockendon, and Prof. J. R. Barber. 


\section{REFERENCES}

[1] L. Afferrante and M. Ciavarella, The thermoelastic Aldo contact model with frictional heating, J. Mech. Phys. Solids, 52 (2004), pp. 617-640, https://doi.org/10.1016/ S0022-5096(03)00116-9.

[2] A. Akande, A Model for Thermoelastic Contact Oscillations, Master's thesis, University of Oxford, Oxford, UK, 2016.

[3] A. E. Anderson And R. A. Knapp, Hot spotting in automotive friction systems, Wear, 135 (1990), pp. 319-337, https://doi.org/10.1016/0043-1648(90)90034-8.

[4] J. F. Archard, Contact and rubbing of flat surfaces, J. Appl. Phys., 24 (1953), pp. 981-988, https://doi.org/10.1063/1.1721448.

[5] D. R. AtтheY, A finite difference scheme for melting problems, IMA J. Appl. Math, 13 (1974), pp. 353-366, https://doi.org/10.1093/imamat/13.3.353.

[6] J. R. BARBeR, Thermoelastic instabilities in the sliding of conforming solids, R. Soc. Proc. A Math. Phys. Eng. Sci., 312 (1969), pp. 381-394, https://doi.org/10.1098/rspa.1969.0165.

[7] M. Ciavarella, L. Johansson, L. Afferrante, A. Klarbring, and J. R. Barber, Interaction of thermal contact resistance and frictional heating in thermoelastic instability, Int. J. Solids Struct., 40 (2003), pp. 5583-5597, https://doi.org/10.1016/S0020-7683(03)00313-5.

[8] M. Di Bernardo, C. J. Budd, A. R. Champneys, and P. Kowalczyk, Piecewise-Smooth Dynamical Systems: Theory and Applications, Springer, London, 2008, https://doi.org/ 10.1007/978-1-84628-708-4.

[9] M. di Bernardo, C. J. Budd, A. R. Champneys, P. Kowalczyk, A. B. Nordmark, G. O. Tost, And P. T. PIIroinen, Bifurcations in nonsmooth dynamical systems, SIAM Rev., 50 (2008), pp. 629-701, https://doi.org/10.1137/050625060.

[10] A. F. Filippov, Differential Equations with Discontinuous Righthand Sides: Control Systems, Kluwer, Dordrecht, the Netherlands, 1988, https://doi.org/10.1007/978-94-015-7793-9.

[11] P. D. Howell, J. R. BArber, And J. R. OCKendon, Multiple-contact thermoelastic oscillations, J. Thermal Stresses, to appear, https://doi.org/10.1080/01495739.2018.1487268.

[12] R. I. LEINE AND D. H. VAN CAMPEN, Bifurcation phenomena in non-smooth dynamical systems, Eur. J. Mech. A Solids, 25 (2006), pp. 595-616, https://doi.org/10.1016/j.euromechsol. 2006.04.004.

[13] J. R. Ockendon and J. R. BARber, A model for thermoelastic contact oscillations, IMA J. Appl. Math., 81 (2016), pp. 679-687, https://doi.org/10.1093/imamat/hxw002.

[14] F. W. J. Olver And R. Wong, NIST Handbook of Mathematical Functions, Cambridge University Press, Cambridge, 2010, pp. 63-66.

[15] K. Popp And P. Stelter, Stick-slip vibrations and chaos, Philos. Trans. Roy. Soc. A, 332 (1990), pp. 89-105, https://doi.org/10.1098/rsta.1990.0102.

[16] D. E. Stewart, Dynamics with Inequalities: Impacts and Hard Constraints, Other Titles Appl. Math. SIAM, Philadelphia, 2011, https://doi.org/10.1137/1.9781611970715.

[17] R. Szalai, Impact mechanics of elastic structures with point contact, J. Vib. Acoust., 136 (2014), 041002, https://doi.org/10.1115/1.4027242.

[18] R. SzalaI, Modelling elastic structures with strong nonlinearities with application to stick-slip friction, R. Soc. Proc. A Math. Phys. Eng. Sci., 470 (2014), 20130593, https://doi.org/10. 1098/rspa.2013.0593.

[19] Y.-B. Yi, J. R. BARBer, AND P. ZAGRodZKI, Eigenvalue solution of thermoelastic instability problems using Fourier reduction, R. Soc. Proc. A Math. Phys. Eng. Sci., 456 (2000), pp. 2799-2821, https://doi.org/10.1098/rspa.2000.0641.

[20] Y. Yoshitake And A. SuEOKa, Forced Self-Excited Vibration with Dry Friction, in Applied Nonlinear Dynamics and Chaos of Mechanical Systems with Discontinuities, World Scientific, Singapore, 2000, pp. 237-259, https://doi.org/10.1142/9789812796301_0010.

Copyright (C) by SIAM. Unauthorized reproduction of this article is prohibited. 